\title{
The effect of tocilizumab on mortality in hospitalized patients with COVID-19: a meta-analysis of randomized controlled trials
}

\author{
Chia Siang Kow ${ }^{1}$ (D) $\cdot$ Syed Shahzad Hasan ${ }^{2,3}$
}

Received: 27 October 2020 / Accepted: 8 January 2021 / Published online: 2 February 2021

(C) The Author(s), under exclusive licence to Springer-Verlag GmbH, DE part of Springer Nature 2021

\begin{abstract}
Objective We aimed to perform a meta-analysis of randomized controlled trials (RCTs) to summarize the overall effect of tocilizumab on the risk of mortality among patients with coronavirus disease 2019 (COVID-19).

Methods We systematically searched PubMed, Cochrane Central Register of Controlled Trials, Google Scholar, and medRxiv (preprint repository) databases (up to 7 January 2021). Pooled effect sizes with 95\% confidence interval (CI) were generated using random-effects and inverse variance heterogeneity models. The risk of bias of the included RCTs was appraised using version 2 of the Cochrane risk-of-bias tool for randomized trials.

Results Six RCTs were included: two trials with an overall low risk of bias and four trials had some concerns regarding the overall risk of bias. Our meta-analysis did not find significant mortality benefits with the use of tocilizumab among patients with COVID-19 relative to non-use of tocilizumab (pooled hazard ratio $=0.83 ; 95 \%$ CI $0.66-1.05, n=2,057$ ). Interestingly, the estimated effect of tocilizumab on the composite endpoint of requirement for mechanical ventilation and/or all-cause mortality indicated clinical benefits, with some evidence against our model hypothesis of no significant effect at the current sample size (pooled hazard ratio $=0.62 ; 95 \%$ CI $0.42-0.91, n=749$ ).

Conclusion Despite no clear mortality benefits in hospitalized patients with COVID-19, tocilizumab appears to reduce the likelihood of progression to mechanical ventilation.
\end{abstract}

Keywords Bias $\cdot$ Clinical trial $\cdot$ COVID-19 $\cdot$ Mortality $\cdot$ Tocilizumab

\section{Introduction}

Tocilizumab is a humanized monoclonal antibody that is directed against the interleukin-6 (IL-6) receptor, wherein IL-6 is considered a key cytokine that involves in the cytokine storm associated with coronavirus disease 2019 (COVID19), characterized by increased alveolar-capillary blood-gas exchange dysfunction, impaired oxygen diffusion, which could eventually lead to pulmonary fibrosis and organ failure [1]. Therefore, there have been attempts to repurpose

Chia Siang Kow

chiasiang_93@hotmail.com

1 School of Postgraduate Studies, International Medical University, Kuala Lumpur, Malaysia

2 School of Applied Sciences, University of Huddersfield, Huddersfield, United Kingdom

3 School of Biomedical Sciences \& Pharmacy, University of Newcastle, Callaghan, Australia tocilizumab for the treatment of patients with COVID-19, especially those who are severely ill and thus presumed to have developed a cytokine storm [2].

Indeed, positive outcomes have been documented with the use of tocilizumab from the retrospective studies among patients with COVID-19. The recent systemic review and metaanalysis of retrospective studies by Zhao et al. [3] have summarized the observational evidence of tocilizumab for the treatment of patients with COVID-19. The meta-analysis, which included 13 retrospective studies, reported that, compared with standard treatment, the use of tocilizumab was associated with significantly reduced odds of mortality (odds ratio $=0.44 ; 95 \%$ confidence interval $0.36-0.55$ ).

Nevertheless, because studies with retrospective designs could introduce biases that may confound the findings, the reported mortality benefits with tocilizumab in patients with COVID-19 must be cautiously interpreted. There have been several randomized controlled trials that reported the effect of tocilizumab on the risk of mortality and other clinical outcomes among patients with COVID-19, and we aim to 
perform a meta-analysis of randomized controlled trials to summarize the overall effect of tocilizumab.

\section{Methods}

\section{Search strategy}

We systematically searched PubMed, Cochrane Central Register of Controlled Trials, Google Scholar, and medRxiv (preprint repository) databases, from 1 January 2020 up to 7 January 2021, with the following keywords and their MeSH terms: "COVID-19," "SARS-CoV-2," "novel coronavirus disease," "anti-IL-6 monoclonal antibody," "IL-6 receptor antagonist," "tocilizumab," "atlizumab," "Actemra," "roactemra," and "randomized controlled trial", without language restrictions.

\section{Inclusion and exclusion criteria}

The inclusion criteria for this meta-analysis were randomized controlled trials comparing the clinical efficacy of tocilizumab and its comparators for the treatment of hospitalized patients with COVID-19. We excluded single-arm trials, nonrandomized trials, and trials that did not report mortality nor outcomes in clinical progression. The primary and secondary outcomes of interest were all-cause mortality and the combined endpoint of requirement for mechanical ventilation and/or all-cause mortality, respectively.

\section{Data extraction and quality assessment}

Each included trial was independently evaluated by two authors (CSK and SSH) who then performed data extraction. Data collected included author(s), trial design, country, age of included patients, the proportion of patients who required respiratory support at baseline, baseline median serum IL-6 level, regimen of tocilizumab and comparative agents, primary outcomes, and mortality outcomes, and their measures of effect. Two authors (SSH and FM) assessed the risk of bias of the included trials using version 2 of the Cochrane risk-of-bias tool for randomized trials (RoB 2) [4], which is a standardized method for assessing potential bias in the reports of randomized interventions. RoB 2 is structured into a fixed set of domains of bias, focusing on different aspects of trial design, conduct, and reporting. A proposed judgment regarding the risk of bias arising from each domain is generated by an algorithm, where judgment can be "low" or "high" risk of bias or can express "some concerns."

\section{Data synthesis and analysis}

For the meta-analysis, we used both the random-effects model and inverse variance heterogeneity (IVhet) model to pool adjusted and unadjusted effect measures of mortality of individual trials to produce a pooled estimate along with the associated $95 \%$ confidence interval. We also pooled the adjusted measures of effect of individual trials that reported the combined endpoint of requirement for mechanical ventilation and/or all-cause mortality. The $\mathrm{I}^{2}$ statistic was performed to estimate how much of the total variability in the effect size estimates are due to heterogeneity among the true effects. All analyses were performed using Meta XL, version 5.3 (EpiGear International, Queensland, Australia).

\section{Results}

\section{Study selection and study characteristics}

Our systematic literature search retrieved 294 hits of which 161 were unique. After screening, six randomized controlled trials [5-10] were included, with a total of 1177 patients who were randomized to receive tocilizumab and 880 patients who were randomized to the control group and did not receive tocilizumab. Details of study selection and the flowchart of the review are depicted in Fig. 1.

There were three global trials $[6,9,10]$, whereas the remaining three trials were each from Italy [7], the United States [5], and France [8]. Details of the included trials are shown in Table 1 . The regimen of tocilizumab administered across the six trials [5-10] was slightly different: in the two trials by Stone et al. [5] and Rosas et al. [6], tocilizumab was administered intravenously at $8 \mathrm{mg} / \mathrm{kg}$ (maximum $800 \mathrm{mg}$ ) as a single dose; whereas in the remaining four trials [7-10], the second dose of tocilizumab was permitted after the administration of the first dose intravenously at $8 \mathrm{mg} / \mathrm{kg}$ (maximum $800 \mathrm{mg}$ ). The second dose of tocilizumab was administered routinely and administered at the discretion of the treating clinician in the two trials by Salvarani et al. [7] and The REMAP-CAP Investigators [10] respectively, but was administered only if no clinical improvement in both the two trials by Hermine et al. [8] and Salama et al. [9].

\section{Risk of bias}

The overall risk of bias assessed by RoB 2 is presented in Table 1. The two trials by Rosas et al. [6] and Salama et al. [9] had a low risk of bias in all the domains assessed (randomization, deviations from intervention, missing outcome data, measurement of the outcome, and selection of the reported results), and thus an overall low risk of bias. The remaining four trials had some concerns in the overall risk of bias; the trials by Stone et al. [5] and by The REMAP-CAP Investigators [10] had some concerns related to bias in the domain of randomization (imbalance in the percentage of older patients between the treatment groups) and in the 
Fig. 1 Flow diagram of study selection

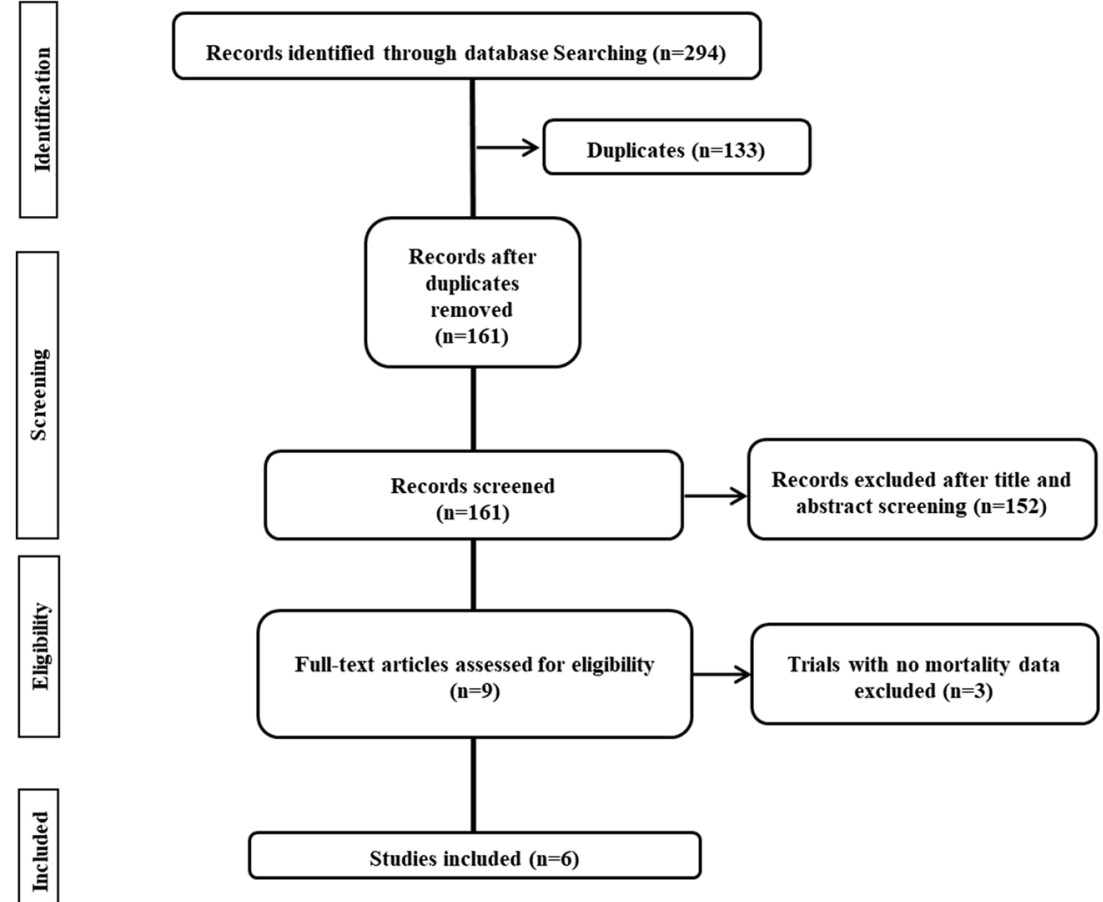

\section{Discussion}

Our meta-analysis of findings from randomized controlled trials, which are the reference standard for studying causal relationships between interventions and outcomes, have proven that the administration of tocilizumab in hospitalized patients with COVID-19 did not reduce the risk of allcause mortality in this patient population, though with significantly reduced likelihood of progression to mechanical ventilation and/or all-cause mortality. It could be hypothesized that patients who had progression to mechanical ventilation despite administration of tocilizumab may consititute a subpopulation of patients with a higher risk of death.

Hence, from our findings, it seems that the inhibition of IL6 alone may not be adequate to mitigate the risk of mortality in patients with COVID-19, at least in certain subpopulation of patients with more severe disease [11]. Indeed, a recent prospective study by Blot et al. [12], which compared the plasma concentrations of IL-6 among patients with COVID-19 associated pneumonia and patients with pneumonia other than COVID-19, reported that the plasma concentrations of IL-6 were significantly lower among the patients with COVID-19 associated pneumonia relative to the patients with pneumonia other than COVID-19 (121.0 pg/mL versus $460.4 \mathrm{pg} / \mathrm{mL})$.

Combination of tocilizumab with a systemic corticosteroid, which diminishes the production of various other inflammatory cytokines in addition to IL- 6 , might be a more useful approach. The subgroup analysis in the trial by Hermine et al. [8] revealed a significantly reduced hazard of mortality in patients with COVID-19 receiving tocilizumab plus ilar pooled effect measures for all the meta-analyses. 


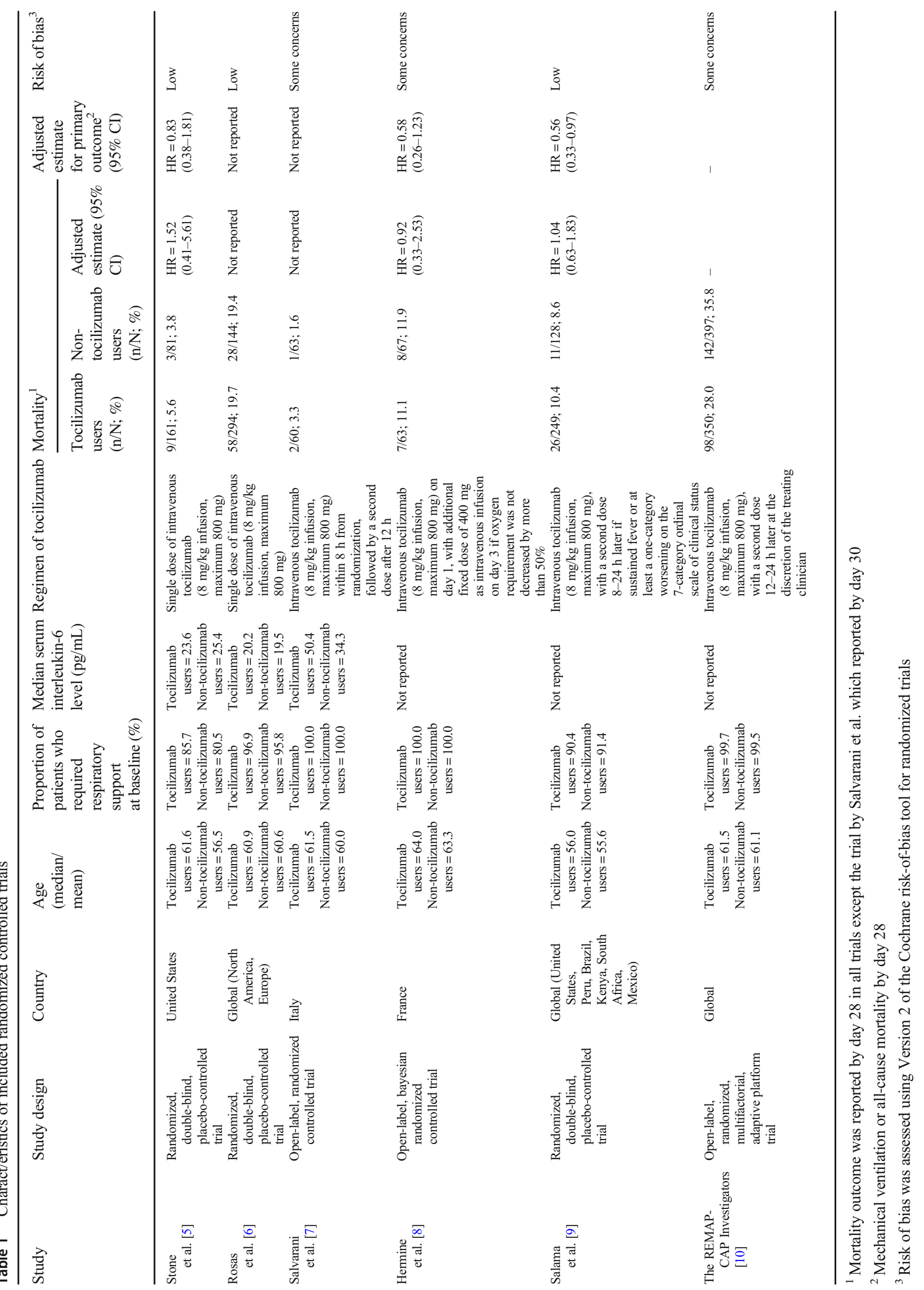


Fig. 2 Forest plot showing the a pooled odds ratio of allcause mortality and $\mathbf{b}$ pooled hazard ratio of requirement for mechanical ventilation and/or allcause mortality between patients with COVID-19 treated with tocilizumab and those not treated with tocilizumab

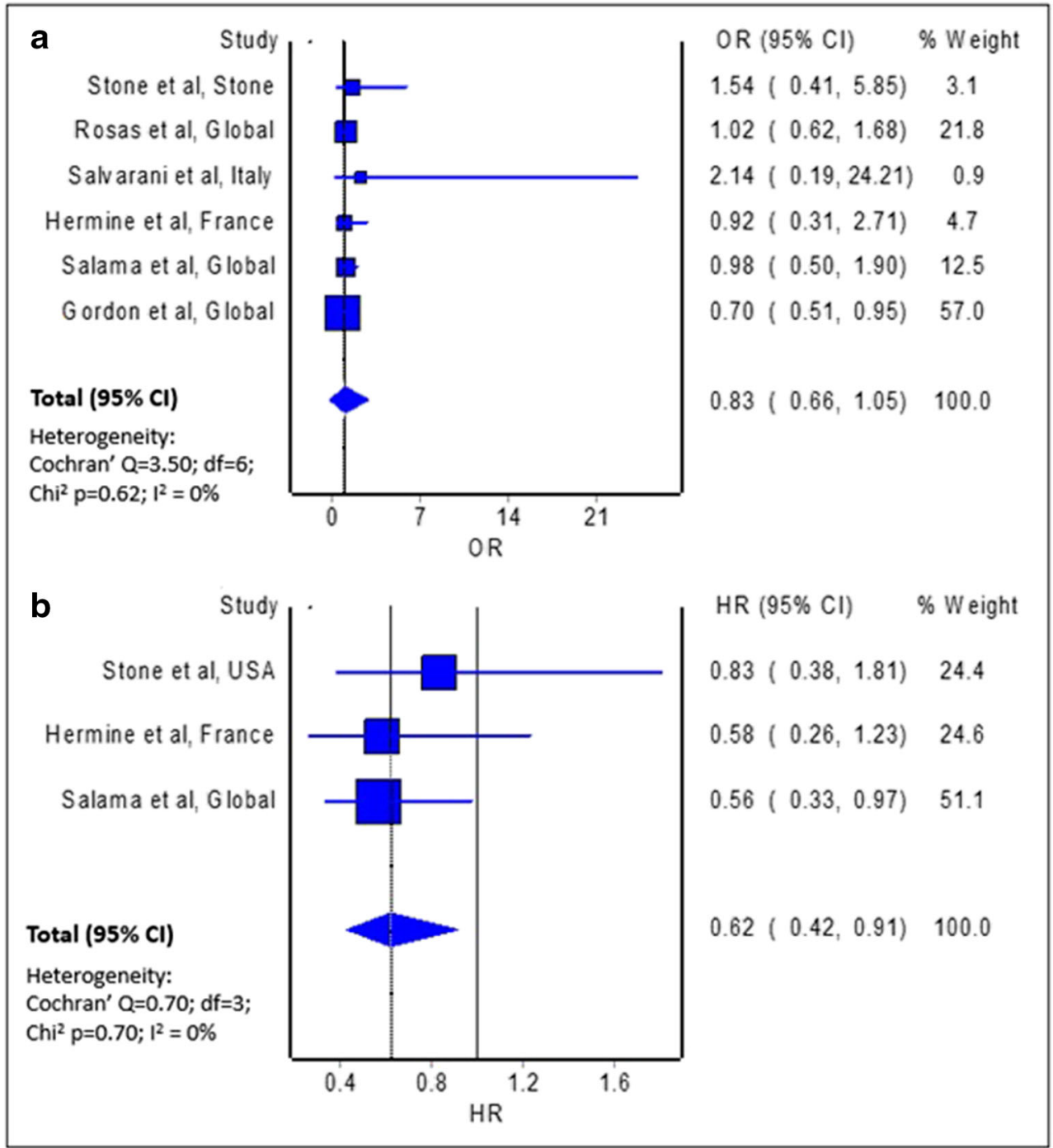

dexamethasone compared to those receiving usual care plus dexamethasone (hazard ratio $=0.13 ; 95 \%$ confidence interval 0.02-0.78). Similarly, the subgroup analysis in the trial by Salama et al. [9] reported a trend toward lower hazard of mortality in patients with COVID-19 who received systemic glucocorticoid use concomitantly or within 7 days of tocilizumab administration (hazard ratio $=0.59 ; 95 \%$ confidence interval 0.34-1.03).

However, it can also be argued that the lack of mortality benefits is associated with relatively low serum IL-6 levels among the participants (median serum IL-6 level $\leq 50 \mathrm{pg} /$ $\mathrm{mL}$ ) across the trials. There are two large studies $[13,14]$ that reported that serum IL-6 level predicts clinical outcomes, such as respiratory failure and death, with an optimal cut-off of $80 \mathrm{pg} / \mathrm{mL}$ and $86 \mathrm{pg} / \mathrm{mL}$, respectively. Therefore, it may be that patients with serum IL- 6 levels of $\geq 80 \mathrm{pg} / \mathrm{mL}$ are more likely to respond to tocilizumab.

The use of tocilizumab in patients with COVID-19 is not without harm. In the trial by Salvarani et al. [7], the proportion of patients randomized to tocilizumab who experienced any adverse events was more than twofold the patients randomized to standard care $(23.3 \%$ versus $11.1 \%$ ). The most frequent adverse event in patients randomized to the tocilizumab arm was increased serum level of alanine aminotransferase $(n=5 / 60$; inclusive of one patient with severe increment). In the trial by Hermine et al. [8], although the proportion of patients randomized to tocilizumab who experienced any adverse effects was less than the patients randomized to usual care (44\% versus $54 \%$ ), it was observed that four patients in the tocilizumab arm developed neutropenia, while none of the patients in the usual care arm developed neutropenia. This was also observed in the trial by Stone et al. [5], where 22 patients $(13.7 \%)$ randomized to tocilizumab experienced neutropenia, while only one patient $(1.2 \%)$ randomized to placebo experienced neutropenia. In the trial by Salvarani et al. [6], bleeding events were more frequent in the tocilizumab group compared to the placebo group (15.3\% versus $11.2 \%)$, 
which may be related to tocilizumab-induced acquired factor XIII deficiency. In the trial by Salama et al. [9], the proportion of patients with adverse events related to the trial drug as determined by the investigator was more frequent in the tocilizumab group compared to the placebo group $(12.8 \%$ versus $3.9 \%)$.

\section{Limitations}

First, our meta-analysis included trials with relatively small scale and with limited heterogeneity across the trials, and therefore the robustness of findings in the individual trials may be limited. However, by combining the results of these trials in a meta-analysis, the robustness of the findings is increased, though more large-scale trials are desirable. Second, we only reported the risk of allcause mortality with and without requirement for mechanical ventilation as our outcomes of interest, since the differential reporting and interpretation of other outcomes across the trials made it difficult to pool these relevant outcomes. Nevertheless, our outcomes of interest, especially the risk of all-cause mortality, has the advantage of being a "hard" endpoint, easy to measure, not readily subject to observer bias, and it represents an important event in patients with COVID-19 who developed a severe illness.

\section{Conclusion}

Our meta-analysis showed that the administration of tocilizumab reduced the likelihood of progression to mechanical ventilation and/or all-cause mortality among hospitalized patients with COVID-19. We did not find a clear benefits in mortality as an endpoint with the administration tocilizumab in hospitalized patients with COVID-19. Our findings should be confirmed with larger randomized controlled trials with longer follow-up. The strategy of administration of tocilizumab can be modified in the future trials to allow its administration exclusively for patients with an increased level of IL-6, in order to determine if tocilizumab influences mortality in this patient population who is theoretically more likely to respond to the effect of tocilizumab. The subgroup analysis that revealed potential mortality benefits with the combination of tocilizumab and a systemic corticosteroid should also be investigated in future trials to determine if such drug combination provides better clinical outcomes than either drug alone. Nevertheless, the administration of tocilizumab in patients with COVID-19 should be accompanied by periodic laboratory monitoring of liver function (to detect significant transaminitis) and complete blood count (to detect neutropenia).
Funding No funding or sponsorship was received for the publication of this article.

\section{Compliance with ethical standards}

Conflicts of interest All named authors declare that they have no potential conflict of interest.

\section{References}

1. Xu X, Han M, Li T et al (2020) Effective treatment of severe COVID-19 patients with tocilizumab. Proc Natl Acad Sci U S A 117(20):10970-10975

2. Singh TU, Parida S, Lingaraju MC, Kesavan M, Kumar D, Singh RK (2020) Drug repurposing approach to fight COVID-19. Pharmacol Rep 72:1479-1508. https://doi.org/10.1007/s43440020-00155-6

3. Zhao M, Lu J, Tang Y, Dai Y, Zhou J Wu Y (2020) Tocilizumab for treating COVID-19: a systemic review and meta-analysis of retrospective studies. Eur J Clin Pharmacol. https://doi.org/ 10.1007/s00228-020-03017-5

4. Sterne JAC, Savović J, Page MJ et al (2019) RoB 2: a revised tool for assessing risk of bias in randomised trials. BMJ. 366:14898

5. Stone JH, Frigault MJ, Serling-Boyd NJ et al (2020) Efficacy of tocilizumab in patients hospitalized with Covid-19. N Engl J Med. https://doi.org/10.1056/NEJMoa2028836

6. Rosas I, Bräu N, Waters M, et al. (2020) Tocilizumab in hospitalized patients with COVID-19 pneumonia. medRxiv. https://doi.org/ $10.1101 / 2020.08 .27 .20183442$

7. Salvarani C, Dolci G, Massari M et al (2020) Effect of tocilizumab vs standard care on clinical worsening in patients hospitalized with COVID-19 pneumonia: a randomized clinical trial. JAMA Intern Med. https://doi.org/10.1001/jamainternmed.2020.6615

8. Hermine O, Mariette X, Tharaux PL et al (2020) Effect of tocilizumab vs usual care in adults hospitalized with COVID-19 and moderate or severe pneumonia: a randomized clinical trial. JAMA Intern Med. https://doi.org/10.1001/jamainternmed.2020.6820

9. Salama C, Han J, Yau L et al (2020) Tocilizumab in patients hospitalized with Covid-19 pneumonia. N Engl J Med. https://doi.org/ 10.1056/NEJMoa2030340

10. The REMAP-CAP Investigators, Gordon AC, Mouncey PR et al (2021) Interleukin-6 receptor antagonists in critically Ill patients with Covid-19 - Preliminary report. medRxiv. https://doi.org/10. 1101/2021.01.07.21249390

11. Chen LYC, Hoiland RL, Stukas S, Wellington CL, Sekhon MS (2020) Confronting the controversy: interleukin-6 and the COVID-19 cytokine storm syndrome. Eur Respir J 56(4):2003006

12. Blot M, Bourredjem A, Binquet C, Piroth L (2020) LYMPHONIE Study Group. Is interleukin 6 the right target in COVID-19 severe pneumonia? Am J Respir Crit Care Med. https://doi.org/10.1164/ rccm.202007-2924LE

13. Herold T, Jurinovic V, Arnreich C et al (2020) Elevated levels of IL-6 and CRP predict the need for mechanical ventilation in COVID-19. J Allergy Clin Immunol 146(1):128-136.e4

14. Laguna-Goya R, Utrero-Rico A, Talayero P et al (2020) IL-6-based mortality risk model for hospitalized patients with COVID-19. J Allergy Clin Immunol 146(4):799-807.e9

Publisher's note Springer Nature remains neutral with regard to jurisdictional claims in published maps and institutional affiliations. 